Faculty of Science

Faculty Publications

This is a post-print version of the following article:

New Insights into Peptide-Silver Nanoparticle Interaction: Deciphering the Role of Cysteine and Lysine in the Peptide Sequence

Horacio Poblete, Anirudh Agarwal, Suma S. Thomas, Cornelia Bohne, Ranjithkumar Ravichandran, Jaywant Phopase, Jeffrey Comer \& Emilio I. Alarcon

January 2016

The final publication is available via Royal Society of Chemistry at:

https://doi.org/10.1021/acs.langmuir.5b03601

Citation for this paper:

Poblete, H., Agarwal, A., Thomas, S. S., Bohne, C., Ravichandran, R., Phopase, J., Comer, J., \& Alarcon, E. I. (2016). New Insights into Peptide-Silver Nanoparticle Interaction: Deciphering the Role of Cysteine and Lysine in the Peptide Sequence. Langmuir, 32, 265-273. https://doi.org/10.1021/acs.langmuir.5b03601. 


\title{
New Insights on Peptide-Silver Nanoparticle Interaction: Deciphering the Role of Cysteine and Lysine in the Peptide Sequence
}

\author{
Horacio Poblete, ${ }^{\mathrm{a}}$ Anirudh Agarwal, ${ }^{\mathrm{b}}$ Suma S. Thomas, ${ }^{\mathrm{c}}$ Cornelia Bohne, ${ }^{\mathrm{c}}$ Ranjithkumar Ravichandran, \\ Jaywant Phospase, ${ }^{\mathrm{d}}$ Jeffrey Comer, ${ }^{\mathrm{a}}$ and Emilio I. Alarcon ${ }^{\mathrm{b}, \mathrm{e}^{*}}$
}

${ }^{a}$ Institute of Computational Comparative Medicine, Nanotechnology Innovation Center of Kansas State, and Department of Anatomy and Physiology, Kansas State University, Manhattan, Kansas, 66506-5802. 'bio-nanomaterials Chemistry and Engineering Laboratory, Cardiac Surgery Research, University of Ottawa Heart Institute, Ottawa, ON, Canada. ${ }^{c}$ Department of Chemistry, University of Victoria, Victoria, BC, Canada. ${ }^{\mathrm{d} D e p a r t m e n t}$ of Physics, Chemistry and Biology, Linköping University, SE 58183 Linköping, Sweden. eDepartment of Biochemistry, Microbiology and Immunology, Faculty of Medicine, University of Ottawa, ON, Canada.

Supporting Information

ABSTRACT: We studied the interaction of four new penta-peptides with spherical silver nanoparticles. Our findings indicate that the combination of the thiol in Cys and amines in Lys/Arg residues are critical to providing stable protection for the silver surface. Molecular simulation reveals the atomic scale interactions that underlie the observed stabilizing effect of these peptides, while yielding qualitative agreement with experiment for ranking the affinity of the four pentapeptides for the silver surface.

\section{- INTRODUCTION}

The interaction between proteins and silver nanoparticles (AgNPs) has been demonstrated to play a pivotal role in the nanomaterial's biocompatibility, and ultimately, its antimicrobial performance. ${ }^{1-5}$ Proteins, ${ }^{2,3,6,7}$ peptides, ${ }^{4,8}$ and free amino acids ${ }^{9}$ can be used to control the structure of AgNPs during synthesis and improve their stability under a variety of conditions, and similar techniques have been applied to other metals, such as gold. ${ }^{10}$ However, the mechanism(s) that underlie such stabilization as well as the exact role of various amino acid moieties remains elusive. The binding affinity of a given peptide for particular crystal facets of the metal appear to mirror the peptide's ability to control nanoparticle synthesis and enhance stability. ${ }^{11,12}$ Thus, understanding peptide binding to Ag surfaces may yield insight on peptide-mediated growth and stabilization of AgNPs. Both experiment and molecular simulation have revealed ${ }^{13,14}$ atomic-level details of binding between peptides and Ag surfaces. In particular, simulations predict high affinities of Arg, Cys, and Met residues for the $\operatorname{Ag}\{111\}$ facet. However, cooperative effects over larger portions of the peptide sequence have long been known to be essential in determining affinity for metal surfaces, ${ }^{15,16}$ and peptides selective for Ag surfaces ${ }^{17}$ further demonstrate that the affinity is not determined solely by individual amino acids, but also by the conformational statistics of the peptide as determined by its sequence. ${ }^{18}$
Furthermore, there is increasing interest in better understanding the biological impact of silver nanoparticles, resulting in an escalating number of proof-of-concept toxicology investigations, overlooking the key role of the surface composition on biological activity, and the long-term effects of AgNPs. ${ }^{19}$ Thus, for example, despite the fact that Cys has been experimentally identified as a key residue in the interaction of proteins with metallic silver, the precise role of this amino acid remains unclear. ${ }^{20} \mathrm{Re}-$ search by members of our team using a capping agentfriendly synthetic route for the preparation of AgNPs, has revealed that lysine (Lys) groups in human serum albumin are key in stabilizing AgNPs. ${ }^{2}$ More recently, we have also found that adding a terminal Cys residue to the LL37 peptide improves its affinity for AgNPs. ${ }^{4}$ This is consistent with the high affinity of Cys for AgNPs, which has been used for Cys detection in biological fluids. ${ }^{21}$ Better understanding of the mechanism of interaction will provide valuable synthetic tools to further advance the design and engineering of new materials for biomedical applications. Thus, in the present work, we have combined experiments with molecular simulation to study the poststabilization effect and synthesis of AgNPs by using four different penta-peptides that were specifically designed to elucidate the relevance of thiol and amine moieties as capping agents for AgNPs. In designing the peptides, we have used a non-charged spacer between the amino terminal and the center aminoacid; Leucine. Thus, the core structure of the peptide comprised X'LX"RS, where the 
aminoacid X' could be Cys or Serine, while X" could be Lysine or Phenylalanine. The terminal RS provided a zwiterionic pair conferring aqueous solubility.

\section{- MATERIAL AND METHODS}

\section{Chemicals and reagents}

Silver nitrate $\left(\mathrm{AgNO}_{3}\right)$, trisodium citrate, 2-hydroxy-1[4-(2-hydroxyethoxy)phenyl]- 2-methyl-1-propanone (I$2959)$, sodium nitrate, and dimethyl sulfoxide ( $>99 \%)$ were purchased from Sigma-Aldrich. Otherwise indicated all aqueous solutions were prepared using Milli-Q water $(18.2 \mathrm{M} \Omega \cdot \mathrm{cm})$.

\section{Synthesis of penta-peptides}

Peptides with sequences (CLKRS, SKFKS, SLFRS, SLKRS, CLFRS) were synthesized on a Symphony automated peptide synthesizer (Protein Technologies Inc., Tucson, AZ, U.S.A.) using standard fluorenylmethoxycarbonyl (Fmoc) chemistry. HCTU (ChemPep Inc., Wellington, FL, USA) was used as the activating reagent. In a $50 \mu \mathrm{mol}$ scale synthesis, Fmoc-L-SER (TBU)-PEGPS resin (Applied Biosystems, Life Technologies Europe BV, Sweden) was used along with four-fold excess of amino acids in each coupling. The resulting peptides were cleaved from the resin by treatment with a mixture of trifluoroacetic acid (TFA), water and triisopropylsilane (TIS) $(95: 2.5: 2.5 \mathrm{v} / \mathrm{v} ; 10 \mathrm{~mL}$ per gram of polymer) for 2 $\mathrm{h}$ at ambient temperature. They were then filtered and the TFA evaporated. Afterward, the peptides were precipitated by the addition of cold diethyl ether, centrifuged and lyophilized. Purification was done using reversed-phase HPLC on a semi-preparative C-18 column (Grace Vydac, Helsingborg, Sweden) and peptide identity was confirmed by their MALDI-TOF spectra (Voyager-DE TM STR Biospectrometry ${ }^{\mathrm{TM}}$ Workstation, PerSeptive Biosystems) (607.0097, 596.6995, 609.1758, 591.0264, 626.6903 for each peptide respectively).

\section{Synthesis of citrate@AgNPs}

Citrate@AgNPs were prepared by methods similar to that described in the literature. ${ }^{3,22-24}$ Briefly, a deoxygenated (30 min $\mathrm{N}_{2}$ ) aqueous solution containing $0.2 \mathrm{mM}$ $\mathrm{AgNO}_{3}, 0.2 \mathrm{mM} \mathrm{I}-2959$, and $1.0 \mathrm{mM}$ sodium citrate was irradiated with UVA light (8 lamps, in a Luzchem LZC-4 photoreactor at $25.0 \pm 0.5^{\circ} \mathrm{C}$ ) for $30 \mathrm{~min}$. The reaction evolution was monitored at the plasmon absorption band $(397 \mathrm{~nm})$. Absorbance spectra were recorded in a Libra S50 UV-Vis spectrophotometer (Biochrom, Cambridge, $\mathrm{UK}$ ) at room temperature, employing $1.0 \mathrm{~cm}$ pathlength cuvettes.

\section{Transmission electron microscopy (TEM) images}

Samples for electron microscopy were prepared by delivering $\sim 5.0 \mu 1$ of solution to carbon-coated copper grids
(400 mesh) and dried in a vacuum system for three days. Electron microscopy images were taken in a JSM-7500F FESEM from JEOL Inc., operating in the transmission mode (TEM) at $15 \mathrm{kV}$. Nanoparticle mean size was calculated from TEM imaging by using ImageJ software ${ }^{12}$ from measuring $>400$ individual particles from different areas in the grid.

\section{Effect of penta-peptides on AgNPs}

The effect of the peptides was assessed by adding microliter volumes of stock solutions of the peptides, in DMSO $(1.0 \mathrm{mM})$, to freshly prepared citrate capped AgNPs. The addition of the peptides was carried out at room temperature and in blind experiments. The absorbance between 350-800 $\mathrm{nm}$ measured in a Libra S50 UVVis spectrophotometer (Biochrom, Cambridge, UK) at room temperature, employing $1.0 \mathrm{~cm}$ pathlength plastic cuvettes. Experiments were carried out in duplicate for four different batches of AgNPs to minimize batch-tobatch variability. Control experiments adding DMSO only did not show any changes in the surface plasmon band of the AgNPs. In all cases $\mathrm{pH}$ of the solution was found in the range of 5.0-5.5.

\section{Stopped-flow measurements for CLKRS binding}

The binding dynamics studies were performed on an Applied Photophysics SX20 stopped-flow system in the absorbance mode for the measurements of the kinetics over $20 \mathrm{~s}$. Two solutions of CLKRS having concentrations of 1.0 and $2.0 \mu \mathrm{M}$ were prepared in deionized water $(\geq 17.8 \mathrm{M} \Omega \mathrm{cm}$ ) from a stock solution of CLKRS in DMSO. Control solutions were also prepared with equal concentrations of DMSO in water without the peptide. The samples were mixed in a 1:1 volume ratio in the mixing chamber that halved the initial concentrations of the samples that are mixed. The temperature of the samples was kept at $20{ }^{\circ} \mathrm{C}$ throughout the experiment. The pathlength of the light through the sample was $2 \mathrm{~mm}$.

The absorbance kinetics for the mixing of AgNP with CLKRS was recorded on stopped-flow at $400 \mathrm{~nm}$ and 450 $\mathrm{nm}$ where the absorbance shows a decrease and an increase respectively. For each concentration of CLKRS and the monitoring wavelength, 16 traces were collected There is a progressive increase in the initial absorbance value for a particular concentration of CLKRS possibly due to aggregation of NPs and thus scattering of light. Figure S4 shows the 16 stopped-flow traces obtained for each experiment.

A Cary 100 spectrometer from Varian was used to collect the kinetics over 50 min using the same stock solutions as for the stopped-flow experiments. Both kinetic experiments were performed on the same day.

\section{Generation of atomistic models}


The molecular structures of all the penta-peptides were constructed and optimized using Chimera ${ }^{25}$ software. The $\mathrm{Ag}\{111\}$ surface was constructed with a lattice parameter of $4.165 \AA$ according to the model of Hughes et al., ${ }^{13}$ which is the product of extensive quantum mechanical calculations. This model includes charged virtual particles rigidly bonded to each silver atom to model polarizability, ${ }^{26}$ as well as virtual interaction sites on the surface that ensure adsorption is most favorable directly atop surface silver atoms and not between them. All silver atoms carried charges of $-0.308 e$, while the rigidly attached virtual particle carried the opposite charge. Water molecules were placed above and below the $\mathrm{Ag}\{111\}$ surface giving equilibrium height of $\sim 4.8 \mathrm{~nm}$ along the $z$ axis. The simulation box was periodic along all three axes. The size of the simulation box in the $x y$ plane was chosen to form supercells of the exposed crystal facets, giving a surface area of $3.7 \mathrm{~nm}^{2}$. All the systems were assembled using VMD 1.9.2 software. ${ }^{27}$

\section{Molecular Dynamics Simulations}

All simulations were executed in the molecular dynamics software NAMD $2.10^{28}$ and employed the standard TIP3P water model used by the CHARMM force field. The temperature and pressure were maintained at $300 \mathrm{~K}$ and $101.325 \mathrm{kPa}(1 \mathrm{~atm})$, respectively, by the Langevin thermostat and Langevin piston ${ }^{29}$ methods. The area of the systems was fixed in the $x y$ plane and the Langevin piston acted only along the $z$-axis. A smooth $0.8-0.9 \mathrm{~nm}$ cutoff of van der Waals forces was employed. Electrostatic interactions were computed via the particle-mesh Ewald algorithm ${ }^{30}$ with a mesh spacing of $<1.2 \AA$. The length of covalent bonds involving hydrogen atoms were constrained $^{31,32}$ to the values prescribed by the CHARMM force field. The equations of motion were integrated using a multiple timestepping scheme ${ }^{33}$ with steps of 2 fs for short- and long-range interactions. For each adsorbate (CLKRS, SLKRS, CLFRS, SLFRS and citrate) system, we performed 2000 steps of energy minimization followed by 100 ps of equilibration before beginning the free-energy calculation. VMD 1.9.2 was used for visualization and molecular renderings. ${ }^{27}$

\section{Free-energy calculations}

Using as a starting point equilibrated systems, the adaptive biasing force ${ }^{34-36}$ method was applied to calculate the free energy as a function of two transition coordinates: $Z=z$ (peptide) $-z(\mathrm{Ag})$ to sample the distance from the peptide to the Ag surface, and $\zeta=z($ res 5$)-z($ res 1$)$ to sample the orientation of the peptide relative to the surface, as was described in detail in Fig 2A,B. The adaptive biasing force method was implemented through the Colvars module ${ }^{37}$ of NAMD $2.10{ }^{28}$ With the goal of further improving sampling, we employed the multiple-walker adaptive biasing force scheme ${ }^{38,39}$ with five walkers and force samples synchronized among the walkers every 5000 simulation steps. The first and second collective variables (Fig $2 \mathrm{a}, \mathrm{b}$ ) were respectively sampled using a single window on the intervals $4 \leq z \leq 16 \AA$ and $-10 \leq \zeta \leq 10 \AA$ for a simulated time of $800 \mathrm{~ns}$ totaled over the five walkers for each peptide. Two additional windows on $-16 \leq \zeta \leq-$ $8 \AA$ and $8 \leq \zeta \leq 16 \AA$ were run for $100 \mathrm{~ns}$ each to complete the sampling. On the other hand, given the reduced conformational phase space of the citrate ion and its smaller size as compared to the peptides, only a single collective variable $(Z)$ was needed to obtain sufficient conformational sampling during $500 \mathrm{~ns}$. Fig. S5 shows the two-dimensional potential of mean force plots for each peptide (A-D) and the conformations of the peptides adsorbed to the $\mathrm{Ag}\{111\}$ surface associated with the global free-energy minima $(\mathrm{E}-\mathrm{H})$.

\section{Synthesis of CLKRS capped AgNPs}

CLKRS capped AgNPs were prepared in a manner similar to that described in the literature for peptide and protein capped AgNPs. ${ }^{2-4,40}$ Briefly, to a deoxygenated (30 $\min \mathrm{N}_{2}$ ) aqueous solution containing $0.2 \mathrm{mM} \mathrm{AgNO}_{3}$ and $0.2 \mathrm{mM}$ I-2959, microliter volumes of CLKRS were added to achieve final concentrations of the peptide between 0.5 and $10 \mu \mathrm{M}$ and the solution was further purged for another $30 \mathrm{~min}$ prior irradiation with UVA light $(8$ lamps, in a Luzchem LZC-4 photoreactor at $25.0 \pm 0.5^{\circ} \mathrm{C}$ ) for $30 \mathrm{~min}$. The reaction evolution was monitored at the plasmon absorption band (400-420 nm). Absorbance spectra were recorded in a Libra S50 UV-Vis spectrophotometer (Biochrom, Cambridge, UK) at room temperature, employing $1.0 \mathrm{~cm}$ pathlength cuvettes. Additionally, $\mathrm{X}$-ray photoelectron spectroscopy measurements were carried out in a Kratos analytical model Axis Ultra DLD, using monochromatic aluminum $\mathrm{K} \alpha \mathrm{X}$-rays at a power of 140 watts, where the presence of metallic silver was observed with its peak centered at binding energies of 366.0 $\mathrm{eV}$ similar to the reported by us in the preparation of collagen capped AgNPs. ${ }^{3}$

\section{- RESULTS AND DISCUSSION}

Synthesis of novel penta-petides. Initially, we assessed the effect of adding peptide concentrations between 0.1 to $5.0 \mu \mathrm{M}$, which is within range where proteins like HSA and type I collagen to provide protection to the surface of spherical AgNPs $\left(4.7 \pm 2.0 \mathrm{~nm}\right.$ in diameter) ${ }^{2,3}$ Fig. 1a shows that CLKRS, CLFRS, and SLKRS modified the surface plasmon band (SPB) of AgNPs, to different extents. Changes in the SPB can be interpreted as a consequence of changes in the nanoparticle surface composition and the data shown in Fig. 1a suggest displacement of citrate from the AgNP's surface by the peptides. Note that no measurable changes in SPB where obtained for peptide SLFRS, see Fig. S1.

Absorption of penta-peptides onto AgNP. When the changes in the absorption intensities at 400 and $430 \mathrm{~nm}$; 
wavelengths that are markedly affected by peptide addition, shown in Fig. 1a are plotted as a function of the peptide concentrations (Fig. 1b): CLKRS is the peptide that most affects the SPB of AgNPs, followed by CLFRS and SLKRS. Interestingly, the hydrodynamic size of AgNPs is increased up to $14 \mathrm{~nm}$ with $1.0 \mu \mathrm{M}$ CLKRS ( $<<0.01$ ), while for the other peptides it remained practically unchanged. We attribute the changes in size to a more efficient replacement of citrate by CLKRS, which could lead to forming a corona arrangement around the nanoparticle surface. a

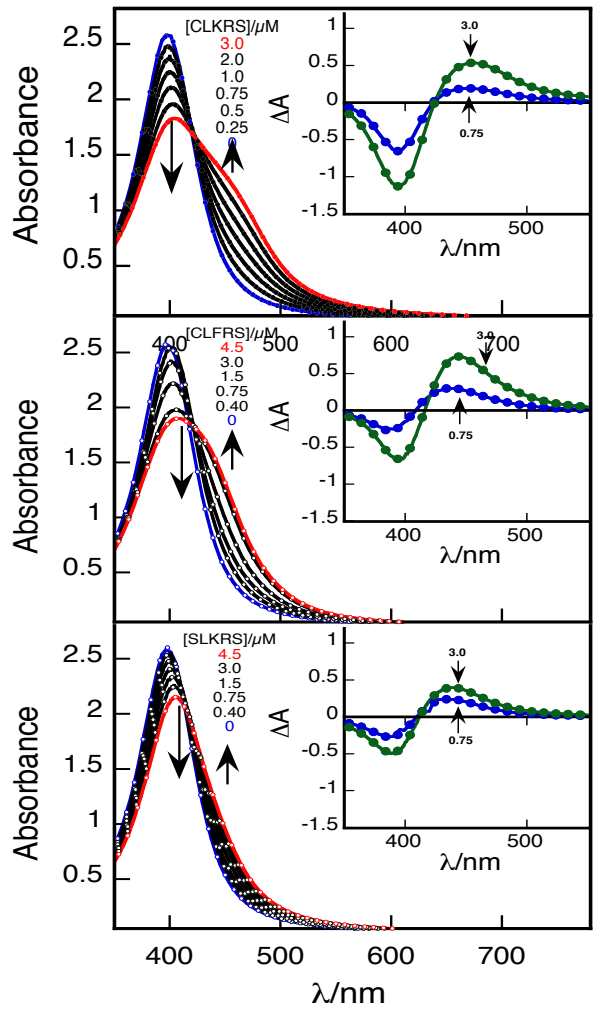

b
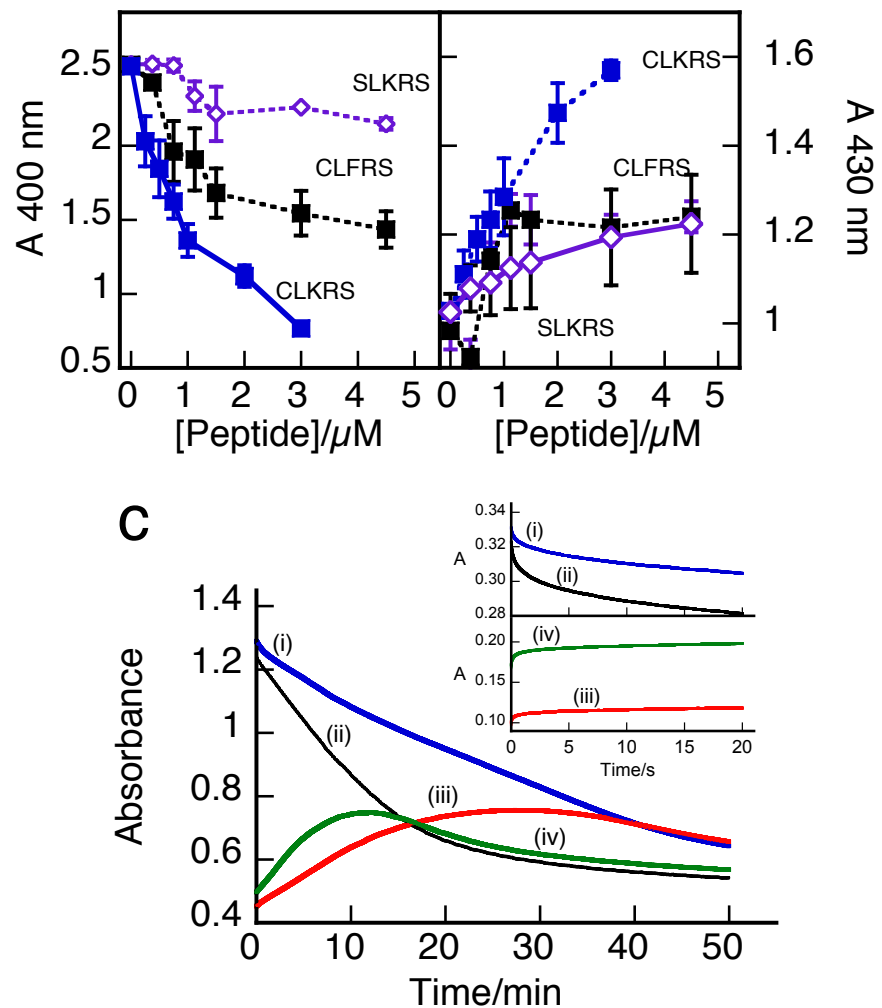

Figure 1. (a) Representative absorption spectra for changes in the surface plasmon band of AgNPs as a function of the peptide concentration for (top to bottom); CLKRS, CLFRS, and SLKRS. Insets show the $\triangle$ Absorbance for the plasmon band measured at different concentrations of the peptides. (b) Changes in plasmon absorption of AgNPs at 400 and $430 \mathrm{~nm}$, left and right, measured at different peptide concentrations. Data shown correspond to average values obtained using five different batches of AgNPs. Blind experiments were carried out in all cases. Error bars correspond to standard error. (c) Average kinetic traces at $400 \mathrm{~nm}([\mathrm{CLKRS}]=0.5 \mu \mathrm{M}$ (i) and $1.0 \mu \mathrm{M}$ (ii)) and $450 \mathrm{~nm}$ ([CLKRS] $=0.5 \mu \mathrm{M}$ (iii) and $1.0 \mu \mathrm{M}$ (iv) collected for $50 \mathrm{~min}$ or as shown in the inset within the first $20 \mathrm{~s}$.

Dynamics of the binding process. Since the extent of SPB modification observed for CLKRS is comparable to the extent obtained using proteins or larger peptides, ${ }^{2,4}$ we decided to further study the mechanistic features of this binding using this peptide. Thus, we assessed the effect of $10 \mathrm{mM}$ sodium citrate, 10 times more citrate that what is shown in Fig. 1a, on the SPB changes upon CLKRS addition, where practically no changes on the SPB were observed, see Fig. S2a. Note that such changes were not observed upon addition of $10 \mathrm{mM} \mathrm{NaNO3}$, which rules out contribution of ionic strength, Fig. S2b. The reversibility of peptide capping was also evaluated. For this purpose, we displaced the pre-bound peptide from the AgNPs by adding higher concentrations of citrate (up to $15 \mathrm{mM}$, which is 7,500 times the peptide concentration, see Fig. $\mathrm{S} 3$ ), where it can be seen that $15 \mathrm{mM}$ sodium citrate is required to replace the bound CLKRS from the AgNP surfaces. Kinetic stopped-flow studies were performed with a focus on establishing the time scales for citrate displacement by CLKRS, where several processes were observed (Fig. 1c). After an immediate increase in absorbance at 400 and $450 \mathrm{~nm}$, a decrease in absorption was observed at $400 \mathrm{~nm}$ over a time scale of $20 \mathrm{~s}$, while an increase occurred at $450 \mathrm{~nm}$ (inset Fig. 1c), which parallels the changes seen for the absorption spectra (Fig. 1a), suggesting that this process corresponds to the replacement of citrate by CLKRS (Fig. S4). The displacement kinetics are not coupled because the apparent half-lives calculated 
for the amplitude at $20 \mathrm{~s}$ are different for the decrease at $400 \mathrm{~nm}$ and the increase at $450 \mathrm{~nm}\left(\left(t_{1 / 2}(400 \mathrm{~nm})=2.8 \pm\right.\right.$ $0.6 \mathrm{~s}$ and $t_{1 / 2}(450 \mathrm{~nm})=0.6 \pm 0.1 \mathrm{~s}$ for [CLKRS] $=0.5$ $\mu \mathrm{M}$, average from individual traces see Table $\mathrm{S} 1$ in the Supporting Information). This result suggests that more than one type of process occurs in the displacement process leading to different changes in the absorption spectra. The absorbance changes continue beyond $20 \mathrm{~s}$, (Fig. 1c) and the rate and time for maximum absorbance depend on the CLKRS concentration. The decrease in absorbance at both wavelengths seen at longer times could be attributed to the formation of less stable CLKRS corona aggregate around the AgNPs. Note that nanoparticle aggregation could be also responsible for changes in the SPB, however under our experimental condition, we did not observe any light scattering, which is characteristic of aggregate formation. The displacement of citrate occurs on the time scale of seconds to minutes, with an initial fast phase followed by slower exchange. This illustrates the complex nature of capping agent replacement, where, for example, corona formation might be taking place. Interestingly, the formation of this corona around the nanoparticle, improves the overall stability of the nanoparticles in higher ionic strength media like $\mathrm{NaCl}$, as seen in Fig. S5.

Overall, the differences observed between the peptides point to different binding modes, and energies, on the nanoparticle surface. To further characterize these binding modes and energies, we carried out molecular dynamics simulations and free-energy calculations.

Molecular simulation and free energy profiles for binding of penta-peptides with AgNPs. Molecular dynamics simulations coupled with free-energy calculation techniques were performed with the aim of unveiling the binding mode of a series of peptides on AgNPs at the atomic level. The low free energy of the $\mathrm{Ag}\{111\}$ facet makes the 111 facet likely to be a significant motif on the AgNPs surfaces. ${ }^{41}$ Thus, we approximated the AgNPs surfaces using the $\operatorname{Ag}\{111\}$ surface model of Hughes et $a l .{ }^{13}$ Fig. $2 \mathrm{~d}$ shows an example of the models of peptides used in the molecular dynamics simulations, which were performed with explicit solvent in atomistic detail. The free-energy calculations were performed using the adaptive biasing force $\mathrm{e}^{35,38}$ technique to determine the free energy as a function of two transition coordinates. The first transition coordinate, $Z$, characterized the distance between the surface and the peptide and was defined as the distance between the center of mass of the peptide and the plane passing through the center of the silver atoms in the first layer of Ag atoms (Fig. 2b). Despite the apparent simplicity of the penta-peptide-Ag $\{111\}$ simulations, the determination of the systems' free-energy as a function of one transition variable $(Z)$ is not altogether trivial, and previous calculations exhibited poor sampling using a conventional single collective variable $(Z)$. In previous work, Replica Exchange with Solvent Tempering ${ }^{42}$ has been shown to yield improved conformational sampling of peptides at inorganic interfaces. Here we use another approach to enhance sampling while also disambiguating the free energies of different conformational states. ${ }^{43} \mathrm{We}$ subjected both $Z$ and a second transition coordinate, $\zeta$, to the multiple-walker adaptive biasing force method. ${ }^{38,39}$ This second coordinate captured the orientation of the peptide relative to the surface and was defined by the difference in the $z$ coordinates between the centers of mass of residues numbers 5 and 1 (Fig. 2c).

Fig. $2 \mathrm{~d}$ shows the two-dimensional free-energy landscape (potential of mean force) calculated for the peptide CLKRS. To clarify the meaning of this plot, Fig. $2 \mathrm{e}-\mathrm{j}$ illustrates typical conformations of the peptide in six different regions of the free-energy landscape. The high-affinity areas (blue) correspond to conformations in which the peptide can interact most strongly with the $\operatorname{Ag}\{111\}$ surface. The global free-energy minimum for CLKRS occurs when $Z \approx 6 \AA$ and $\zeta \approx 2.5 \AA$ (Fig. $2 \mathrm{f}$ ), wherein sidechains of Cys1 and Arg4 make contact with the surface. The broad favorable region near label $\mathrm{H}$ is associated with Cys 1 and Lys 3 side-chains at the surface. Favorability in this region of the 2D potential of mean force is significantly reduced when lysine is changed to phenylalanine (Fig. S6), leading to an overall reduced affinity of CLFRS versus CLKRS (see below). The interaction between the thiol group of Cys1 and $\mathrm{Ag}\{111\}$ appears particularly stabilizing, as most of the lowest free-energy configurations include Cys- $\mathrm{Ag}$ contact (Fig. 2e-i). Interestingly, the marginally favorable region near $Z \approx 12 \AA$ and $\zeta \approx-12 \AA$ corresponds to conformations where the $\mathrm{COO}^{-}$terminus of the peptide binds not to the $\operatorname{Ag}\{111\}$ surface itself, but to the ordered water layer just above. Interaction with the water layer has been observed in previous work using the Hughes et al. force field. ${ }^{13}$ The $2 \mathrm{D}$ potentials of mean force for the other peptides are shown in Fig. S6. 

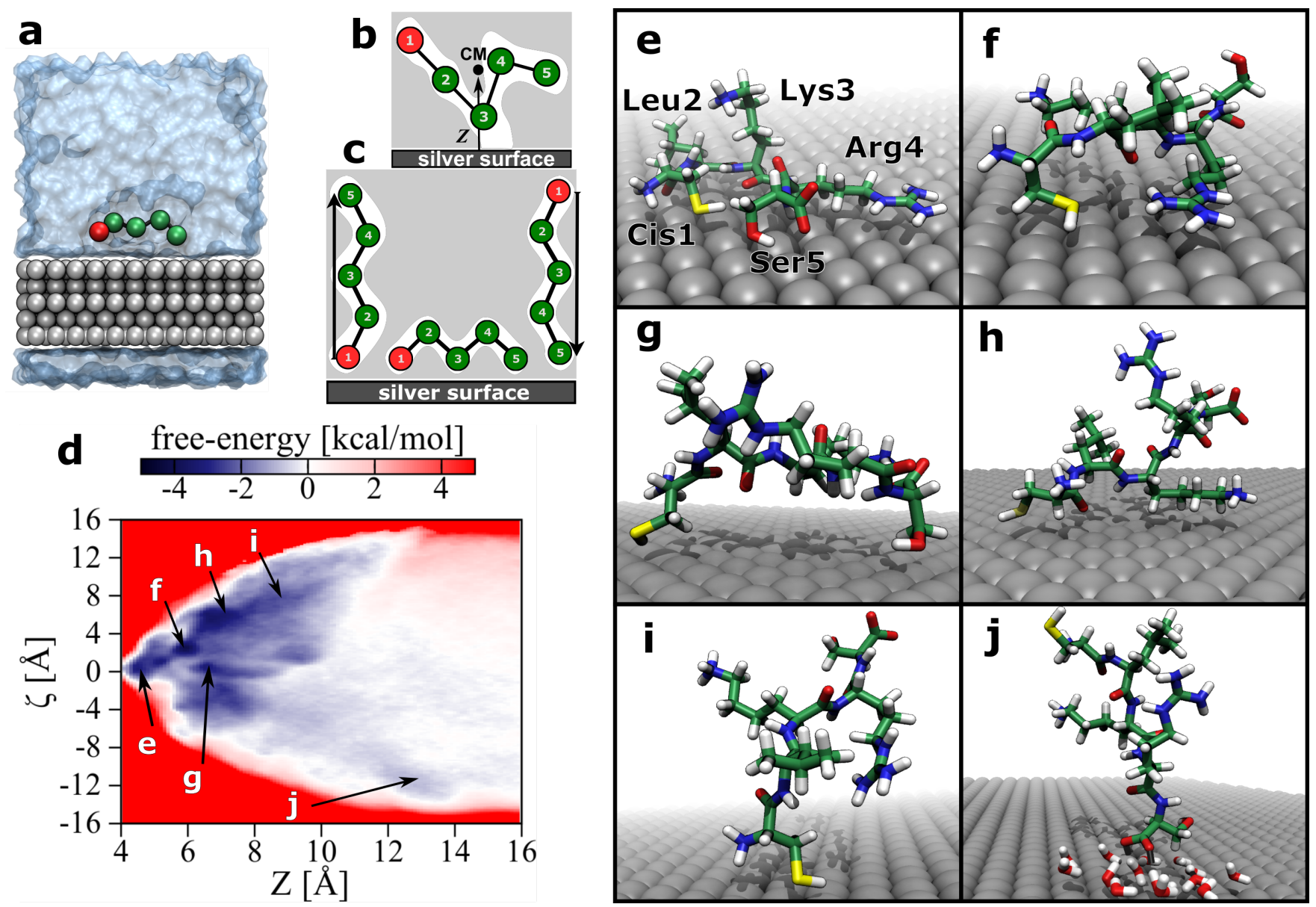

Figure 2. Molecular simulation of peptide adsorption to an $\operatorname{Ag}\{111\}$ surface. (a) Example of the molecular dynamics models. Water is shown as a transparent blue surface while silver atoms are shown as gray spheres. The peptide is represented as green spheres for each residue, with the first residue highlighted in red. For clarity, the atomic details of the peptide are not represented. The additional particles (for polarizability and atop adsorption) of the Hughes et al., model of Ag \{111\} are likewise suppressed. (b) Diagram of the $Z$ transition coordinate, which is the distance between the center of mass of the peptide and the plane passing through the first layer of silver atoms. (c) Second transition coordinate, $\zeta$ : the difference in the $z$ coordinates between the centers of mass of residue 5 and 1. (d) Two-dimensional free-energy landscape for the peptide CLKRS. (e-j) Typical conformations of this peptide corresponding to the letters marked in the free energy plot (Fig 2d).

The 2D potentials of mean force can be integrated to yield more easily interpretable 1D free-energy profiles, which are shown in Figure 3A. For comparison with experiments including sodium citrate, we have also calculated a 1D potential of mean force for citrate ${ }^{3-}$, a citrate ion with all carboxyl groups fully deprotonated. In all cases, the free energy reaches a plateau for distances between the surface and peptide $>13 \AA$, while at short distances, the free energy grows rapidly because the adsorbate (peptide or citrate ${ }^{3-}$ ) is sterically prevented from penetrating the surface. Free-energy minima on the interval $5<Z<7.5 \AA$ are evident for all adsorbates and represent the most favorable distance of the center of mass of the adsorbate from the surface. Strong interaction with the cysteine thiol group favors contact with the surface even when the center of mass of the peptide is relatively far from the surface, as is exemplified in Fig. 2i. These favorable interactions are responsible for the relatively low free energies apparent in Fig. 3a for the cysteine containing peptides CLKRS and CLFRS at distances $Z>8 \AA$, while interactions with the other peptides remain marginal at these distances. The peptides SLKRS and SLFRS, as well as citrate ${ }^{3-}$, exhibit relatively weak adsorption compared to the peptides CLKRS and CLFRS.

To further distill the simulation results, we estimated standard binding free energies in the dilute limit between the adsorbates and AgNPs of sizes similar to those observed in experiment ( $4.7 \mathrm{~nm}$ diameter). Following Shoup and $S z a b o,{ }^{44}$ we calculated the standard equilibrium constant as $\mathrm{K}=\frac{\mathrm{A}}{\mathrm{c}_{0}} \int_{0}^{\mathrm{b}} \mathrm{dZ} \mathrm{e}^{-\beta \mathrm{w}(\mathrm{Z})}$, where $A$ is the area of the nanoparticle, $c_{0}$ is the standard concentration $(1 \mathrm{~mol} / \mathrm{L})$, the limits of the integral define the distance interval where the system is considered bound, $\beta=1 /\left(k_{\mathrm{B}} T\right)$ is the inverse thermal energy, and $w(Z)$ is a free-energy profile like those shown in Fig. 3a. The value $A$ was taken to be that for a spherical nanoparticle with a diameter of $4.7 \mathrm{~nm}$, similar to those observed in the experiments. We chose $b=10 \AA$; however, any reasonable choice of the upper limit of the integral, $b$, would yield essentially identical 
results. ${ }^{45}$ The standard Gibbs free energies for binding each peptide and citrate ${ }^{3-}$ to an AgNP, computed as $\Delta \mathrm{G}^{\circ}=$ $-\mathrm{k}_{\mathrm{B}} \mathrm{T} \ln \mathrm{K}$, are shown in Fig. 3b. CLKRS binds most strongly to the $\operatorname{Ag}\{111\}$ surface, followed by CLFRS. Although the changes in absorbance shown in Figure $1 \mathrm{~b}$ are not rigorously proportional to changes in the surface density of peptide, they provide an approximate indication that the results of the calculations are reasonable. For instance, the calculations give $\Delta \mathrm{G}$ values of -3.39 and $3.68 \mathrm{kcal} / \mathrm{mol}$ for CLFRS and CLKRS, respectively. This implies that the ratio of adsorbed CLFRS to CLKRS

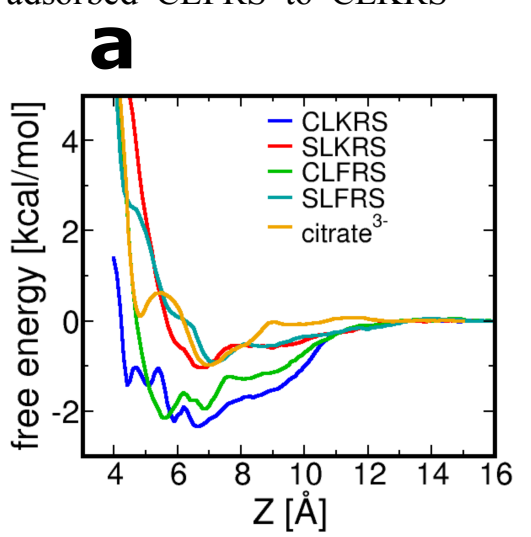

should be $\exp [-\beta(\Delta \mathrm{G}(\mathrm{CLFRS})-\Delta \mathrm{G}(\mathrm{CLKRS}))]=61 \%$ at equal bulk concentrations of peptide in the low surfacecoverage limit. In rough agreement with this ratio, at peptide concentrations of $1 \mu \mathrm{M}$, Figure $1 \mathrm{~b}$ shows that the absorbance at $400 \mathrm{~nm}$ in the presence of CLKRS is roughly $68 \%$ that at in the presence of CLFRS. All peptides except SLFRS show significantly stronger binding than citrate ${ }^{3-}$ , consistent with the displacement of citrate ions by these peptides shown in Fig. 1 and Fig. S1.

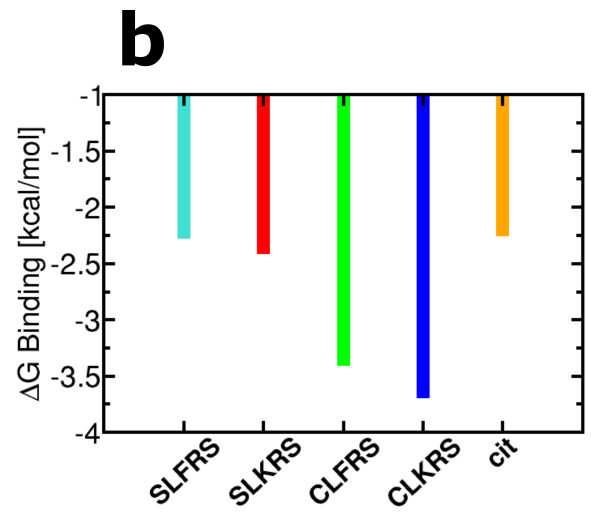

Figure 3. Free-energy of peptide:AgNPs binding from molecular dynamics simulation. (a) Free-energy profiles along the single transition coordinate $Z$ for the citrate ${ }^{-}$ion and the peptides considered in this work. (b) Estimated $\Delta G$ for binding of the peptides or citrate $\mathrm{e}^{-}$ion to a $4.7 \mathrm{~nm}$ diameter AgNPs.

We then explored the ability of the three peptides, to act as capping agents for unprotected AgNPs. To this end, we prepared AgNPs using a photochemical approach we had previously used for the synthesis of biomolecule capped AgNPs. ${ }^{2,4,40}$ This synthetic approach allows the production of unprotected $\mathrm{Ag}$ atoms that will nucleate to form larger nanocrystals. The reduction of ionic silver occurs upon reaction of a photogenerated ketyl radical with $\mathrm{Ag}(\mathrm{I}) .{ }^{23}$ If no protecting agent is added into the mixture, unstable AgNPs are formed which spontaneously aggregate/oxidize. Fig. 4a shows that uniquely CLKRS is capable to produce stable AgNPs. Those particles have a maximum absorption $\approx 415 \mathrm{~nm}$. This maximum shifts to a shorter wavelength when the peptide concentration is increased to $10 \mu \mathrm{M}(401 \mathrm{~nm})$. Fig. $4 \mathrm{~b}$ shows the time profile for AgNPs SPB formation as a function of the irradiation time for various concentrations of CLKRS from 1.0 to $10 \mu \mathrm{M}$. Since a direct comparison between the curves is not possible as they present different absorption maxima, we can a priori infer, from the initial slopes region (see inset Fig. 4b), that AgNPs are formed at similar rates at 2.5 and $5.0 \mu \mathrm{M}$ CLKRS. Synthesis of AgNPs using
CLKRS produces spherical nanoparticles as shown in Fig. 4c. Transmission electron microscopy (TEM) images for the AgNPs prepared using different concentrations of the CLKRS, see Fig. 4c, see Fig. S7 for representative images, indicate that the average diameter for the nanoparticles decreased as the CLKRS concentration increases from 45 to $5.7 \mathrm{~nm}$ for 0.5 and $5.0 \mu \mathrm{M}$ CLKRS, respectively, see inset Fig 4c. This figure also shows the nanoparticle size distribution obtained for CLFRS at 1.0 $\mu \mathrm{M}$, where a broad size distribution is clearly observed. Although the size of AgNPs obtained when using CLKRS does not compare to that obtained for citrate protected AgNPs, inset Fig. $4 c(p<0.0001)$, we observed that the nanoparticle diameter can be finely tuned from 45 to $5.0 \mathrm{~nm}$ by simply varying the peptide concentration from 0.5 to $5.0 \mu \mathrm{M}$. Those changes in size with the peptide concentration can be interpreted in terms of changes in the nanoparticle growing ${ }^{23}$ as similar to the reported by us in the formation of AgNPs using LL37-SH as capping agent. ${ }^{4}$ 

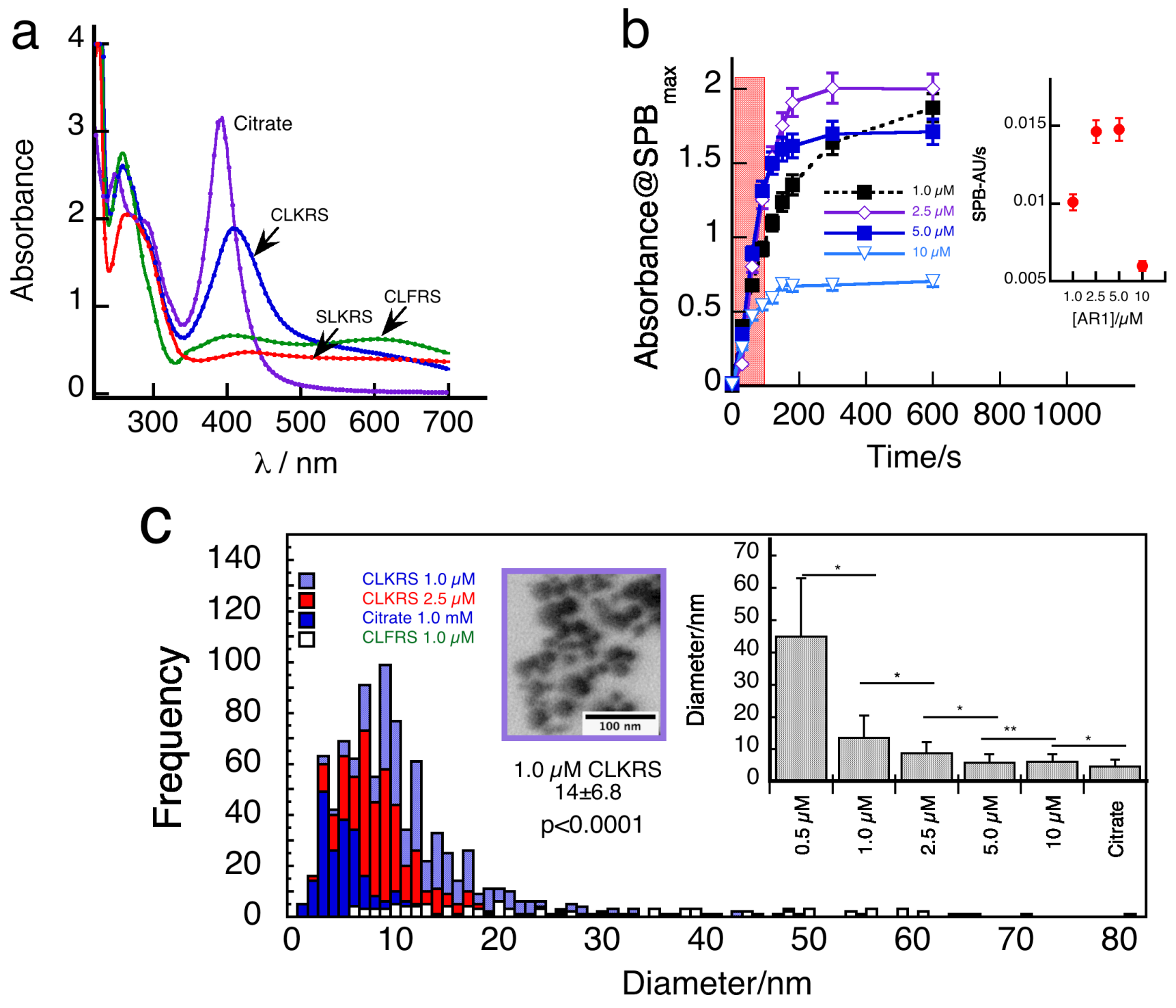

Figure 4. (a) Absorption spectra for AgNPs prepared using sodium citrate, CLKRS, CLFRS or SLKRS as capping agents. Spectra obtained after 10 min UVA irradiation of an aqueous solution containing $0.2 \mathrm{mM} \mathrm{AgNO}_{3}, 0.2 \mathrm{mM}$ I-2959 and the chosen capping agent. (b) Changes on AgNPs surface plasmon band maximum as a function of the irradiation time, obtained using different concentrations of CLKRS. Inset shows the dependence of the initial slope region, highlighted inside a red box in the plot, at different CLKRS concentrations. (c) Size distribution histograms for AgNPs prepared using 1.0 and $2.5 \mu \mathrm{M}$ CLKRS. The histograms obtained using sodium citrate $1.0 \mathrm{mM}$ or CLFRS $1.0 \mu \mathrm{M}$ are also included. Images in the plot correspond to representative TEM images for the samples. Inset: Plot bar for average nanoparticle diameter obtained from TEM measurements for AgNPs prepared using CLKRS $(0.5$ to $10 \mu \mathrm{M})$ and sodium citrate $1.0 \mathrm{mM}$. Average sizes were calculated from measuring $>100$ individual particles in different areas of the grid. Blind samples were used in all cases. Error bars correspond to the standard deviation. Confidence $\mathrm{p}$ values from t-tests are $\mathrm{p}<0.0001(*)$ and $\mathrm{p}>0.1(* *)$.

\section{- CONCLUSION}

We have identified a new high affinity five amino acid sequence for post-stabilization and capping of AgNPs; CLKRS. Further, the peptide can be used as a capping agent for nascent AgNPs; by simply varying the peptide concentration between $0.5-5.0 \mu \mathrm{M}$, one can produce spherical AgNPs with sizes ranging from 45.0 to $5.0 \mathrm{~nm}$. Kinetic studies with CLKRS showed that citrate replacement is complex with more than one species being formed and with kinetic processes occurring in the seconds to minutes time scales. In addition, by using molecular dynamics simulations, we learned that the presence of an amine terminal cysteine plays a pivotal role for the strong interaction of the peptides with the metal surface. However, cooperative effects among the amino acids were also evident; for example, simulations revealed that CLFRS, relative to CLKRS, lacked a particular low free-energy region in configuration space and was associated with the 
reduced efficacy of CLFRS as a capping agent as determined experimentally.

\section{- AUTHOR INFORMATION}

\section{Corresponding Author}

Enquires must be addressed to jeffcomer@ksu.edu and Ealarcon@,ottawaheart.ca

\section{- ASSOCIATED CONTENT}

Supporting Information. Effect of the addition of $3.0 \mu \mathrm{M}$ SLFRS to citrate protected AgNPs; Changes on AgNPs SPB upon 3.0 $\mu \mathrm{M}$ CLKRS measured in the presence of either 1 or $10 \mathrm{mM}$ sodium citrate and sodium nitrate $10 \mathrm{mM}$; Effect of sodium citrate addition on a colloidal solution of CLKRS $2.0 \mu \mathrm{M}$ and citrate capped AgNPs; Stopped-flow traces for the mixing of AgNP with $0.5 \mu \mathrm{M}$ and $1 \mu \mathrm{M}$ of CLKRS recorded at $400 \mathrm{~nm}$ and $450 \mathrm{~nm}$; Effect of $\mathrm{NaCl}$ on AgNPs stability in the presence of $3.0 \mu \mathrm{M}$ CLKRS; Two-dimensional free-energy landscapes for penta-peptides CLKRS, SLKRS, CLFRS and SLFRS. Typical conformations associated with minimum free energy for CLKRS, SLKRS, CLFRS and SLFRS; Representative TEM images for AgNPs prepared using different concentrations of CLKRS or $1.0 \mathrm{mM}$ citrate; and Apparent half-life values obtained from the kinetic traces at different concentrations of CLKRS monitored at 400 and $450 \mathrm{~nm}$. This material is available free of charge via the Internet at http://pubs.acs.org.

\section{- ACKNOWLEDGMENTS}

No competing financial interests have been declared. The authors thank Zak Hughes for providing the Gromacs parameter files for the AgNPs force field. This work was supported by NSERC Discovery (EIA) and University of Ottawa Heart Institute (EIA). This work was also supported by the Project FONDECYT 3140288 (HP). HP and JC were partially supported by the Kansas Bioscience Authority funds to the Institute of Computational Comparative Medicine (ICCM) at Kansas State University and to the Nanotechnology Innovation Center of Kansas State University (NICKS). The Kansas State University Global Food Systems Innovation Program (383GFS) provided further support (HP and JC). Computing for this project was performed on the Beocat Research Cluster at Kansas State University, which is funded in part by NSF grants CNS-1006860, EPS1006860, and EPS-0919443. Additional computer time was generously provided by the Extreme Science and Engineering Discovery Environment (XSEDE) under request TGMCB150036. XSEDE is supported by National Science Foundation grant number ACI-1053575. The work at the University of Victoria was supported by an NSERC Discovery Grant (RGPIN-121389-2012). The work at Linköping University was supported by Swedish Research Council (dnr 621-2012-4286).

\section{- REFERENCES}

(1) Niemeyer, C. M. Nanoparticles, Proteins, and Nucleic Acids: Biotechnology Meets Materials Science. Angew. Chem. Int. Ed. 2001, 40 (22), 4128-4158.

(2) Alarcon, E. I.; Bueno-Alejo, C. J.; Noel, C. W.; Stamplecoskie, K. G.; Pacioni, N. L.; Poblete, H.; Scaiano, J. C. Human Serum Albumin as Protecting Agent of Silver Nanoparticles: Role of the Protein Conformation and Amine Groups in the Nanoparticle Stabilization. J. Nanoparticle Res. 2013, 15 (1), 1-14.

(3) Alarcon, E. I.; Udekwu, K.; Skog, M.; Pacioni, N. L.; Stamplecoskie, K. G.; González-Béjar, M.; Polisetti, N.; Wickham, A.; RichterDahlfors, A.; Griffith, M.; et al. The Biocompatibility and Antibacterial Properties of Collagen-Stabilized, Photochemically Prepared Silver Nanoparticles. Biomaterials 2012, 33 (19), 4947-4956.

(4) Vignoni, M.; de Alwis Weerasekera, H.; Simpson, M. J.; Phopase, J.; Mah, T.-F.; Griffith, M.; Alarcon, E. I.; Scaiano, J. C. LL37 Peptide@ Silver Nanoparticles: Combining the Best of the Two Worlds for Skin Infection Control. Nanoscale 2014, 6 (11), 5725-5728.

(5) Monteiro-Riviere, N. A.; Samberg, M. E.; Oldenburg, S. J.; Riviere, J. E. Protein Binding Modulates the Cellular Uptake of Silver Nanoparticles into Human Cells: Implications for in Vitro to in Vivo Extrapolations? Toxicol. Lett. 2013, 220 (3), 286-293.

(6) Murawala, P.; Phadnis, S. M.; Bhonde, R. R.; Prasad, B. L. V. In Situ Synthesis of Water Dispersible Bovine Serum Albumin Capped Gold and Silver Nanoparticles and Their Cytocompatibility Studies. Colloids Surf. B Biointerfaces 2009, 73 (2), 224-228.

(7) Gebauer, J. S.; Malissek, M.; Simon, S.; Knauer, S. K.; Maskos, M.; Stauber, R. H.; Peukert, W.; Treuel, L. Impact of the NanoparticleProtein Corona on Colloidal Stability and Protein Structure. Langmuir 2012, 28 (25), 9673-9679.

(8) Naik, R. R.; Stringer, S. J.; Agarwal, G.; Jones, S. E.; Stone, M. O. Biomimetic Synthesis and Patterning of Silver Nanoparticles. Nat. Mater. 2002, 1 (3), 169-172.

(9) Koley, P.; Pramanik, A. Nanostructures from Single Amino Acid-Based Molecules: Stability, Fibrillation, Encapsulation, and Fabrication of Silver Nanoparticles. Adv. Funct. Mater. 2011, 21 (21), 4126-4136.

(10) Slocik, J. M.; Stone, M. O.; Naik, R. R. Synthesis of Gold Nanoparticles Using Multifunctional Peptides. Small 2005, 1 (11), 1048-1052.

(11) Zeng, Q.; Jiang, X.; Yu, A.; Lu, G. (Max). Growth Mechanisms of Silver Nanoparticles: A Molecular Dynamics Study. Nanotechnology 2007, 18 (3), 035708

(12) Li, Y.; Tang, Z.; Prasad, P. N.; Knecht, M. R.; Swihart, M. T. Peptide-Mediated Synthesis of Gold Nanoparticles: Effects of Peptide Sequence and Nature of Binding on Physicochemical Properties. Nanoscale 2014, 6 (6), 3165-3172.

(13) Hughes, Z. E.; Wright, L. B.; Walsh, T. R. Biomolecular Adsorption at Aqueous Silver Interfaces: First-Principles Calculations, Polarizable Force-Field Simulations, and Comparisons with Gold. Langmuir 2013, 29 (43), 13217-13229.

(14) Palafox-Hernandez, J. P.; Tang, Z.; Hughes, Z. E.; Li, Y.; Swihart, M. T.; Prasad, P. N.; Walsh, T. R.; Knecht, M. R. Comparative Study of Materials-Binding Peptide Interactions with Gold and Silver Surfaces and Nanostructures: A Thermodynamic Basis for Biological Selectivity of Inorganic Materials. Chem. Mater. 2014, 26 (17), 4960-4969.

(15) Brown, S. Metal-Recognition by Repeating Polypeptides : Abstract : Nature Biotechnology. Nat Biotech 1997, 15 (3), 269-272. 
H.; Naik, R. R.; Knecht, M. R. Crystallographic Recognition Controls Peptide Binding for Bio-Based Nanomaterials. J. Am. Chem. Soc. 2011, 133 (32), 12346-12349.

(17) Hnilova, M.; Liu, X.; Yuca, E.; Jia, C.; Wilson, B.; Karatas, A. Y.; Gresswell, C.; Ohuchi, F.; Kitamura, K.; Tamerler, C. Multifunctional Protein-Enabled Patterning on Arrayed Ferroelectric Materials. ACS Appl. Mater. Interfaces 2012, 4 (4), 1865-1871.

(18) Tang, Z.; Palafox-Hernandez, J. P.; Law, W.-C.; E. Hughes, Z.; Swihart, M. T.; Prasad, P. N.; Knecht, M. R.; Walsh, T. R. Biomolecular Recognition Principles for Bionanocombinatorics: An Integrated Approach To Elucidate Enthalpic and Entropic Factors. ACS Nano 2013, 7 (11), 9632-9646.

(19) de Alwis Weerasekera, H.; Griffith, M.; Alarcon, E. I. Biomedical Uses of Silver Nanoparticles: From Roman Wine Cups to Biomedical Devices. In Silver Nanoparticle Applications; Springer, 2015; pp 93-125.

(20) Eckhardt, S.; Brunetto, P. S.; Gagnon, J.; Priebe, M.; Giese, B.; Fromm, K. M. Nanobio Silver: Its Interactions with Peptides and Bacteria, and Its Uses in Medicine. Chem. Rev. 2013, 113 (7), 4708-4754.

(21) Leesutthiphonchai, W.; Dungchai, W.; Siangproh, W.; Ngamrojnavanich, N.; Chailapakul, O. Selective Determination of Homocysteine Levels in Human Plasma Using a Silver Nanoparticle-Based Colorimetric Assay. Talanta 2011, 85 (2), 870-876.

(22) Jockusch, S.; Landis, M. S.; Freiermuth, B.; Turro, N. J. Photochemistry and Photophysics of $\alpha$-Hydroxy Ketones. Macromolecules 2001 34 (6), 1619-1626.

(23) Scaiano, J. C.; Netto-Ferreira, J. C.; Alarcon, E.; Billone, P.; Alejo, C. J. B.; Crites, C.-O. L.; Decan, M.; Fasciani, C.; González-Béjar, M.; Hallett-Tapley, G.; et al. Tuning Plasmon Transitions and Their Applications in Organic Photochemistry. Pure Appl. Chem. 2011, 83 (4), 913 930.

(24) Stamplecoskie, K. G.; Scaiano, J. C. Light Emitting Diode Irradiation Can Control the Morphology and Optical Properties of Silver Nanoparticles. J. Am. Chem. Soc. 2010, 132 (6), 1825-1827.

(25) Pettersen, E. F.; Goddard, T. D.; Huang, C. C.; Couch, G. S.; Greenblatt, D. M.; Meng, E. C.; Ferrin, T. E. UCSF Chimera-a Visualization System for Exploratory Research and Analysis. J. Comput. Chem. 2004, 25 (13), 1605-1612.

(26) Iori, F.; Corni, S. Including Image Charge Effects in the Molecular Dynamics Simulations of Molecules on Metal Surfaces. J. Comput. Chem. 2008, 29 (10), 1656-1666.

(27) Humphrey, W.; Dalke, A.; Schulten, K. VMD: Visual Molecular Dynamics. J.Mol. Graph. 1996, 14 (1), 33-38.

(28) Phillips, J. C.; Braun, R.; Wang, W.; Gumbart, J.; Tajkhorshid, E.; Villa, E.; Chipot, C.; Skeel, R. D.; Kale, L.; Schulten, K. Scalable Molecular Dynamics with NAMD. J. Comput. Chem. 2005, 26 (16), 17811802 .

(29) Feller, S. E.; Zhang, Y.; Pastor, R. W.; Brooks, B. R. Constant Pressure Molecular Dynamics Simulation: The Langevin Piston Method. J. Chem. Phys. 1995, 103, 4613 .

(30) Darden, T.; York, D.; Pedersen, L. Particle Mesh Ewald: An N. Log (N) Method for Ewald Sums in Large Systems. J. Chem. Phys. 1993, 98 (12), 10089-10092.
(31) Andersen,H.C. RATTLE: A "Velocity" Version of the SHAKE Algorithm for Molecular Dynamics Calculations. J. Comput. Phys. 1983 $52(1), 24-34$

(32) SETTLE, M. S. K. P. An Analytical Version of the SHAKE and RATTLE Algorithm for Rigid Water Molecules. J Comput Chem 1992, 13, 952-962.

(33) Tuckerman, M.; Berne, B. J.; Martyna, G. J. Reversible Multiple Time Scale Molecular Dynamics. J. Chem. Phys. 1992, 97 (3), 1990-2001

(34) Comer, J.; Gumbart, J. C.; Hénin, J.; Lelièvre, T.; Pohorille, A.; Chipot, C. The Adaptive Biasing Force Method: Everything You Always Wanted to Know but Were Afraid to Ask. J. Phys. Chem. B 2014, 119 (3), 1129-1151.

(35) Darve, E.; Pohorille, A. Calculating Free Energies Using Average Force. J. Chem. Phys. 2001, 115 (20), 9169-9183.

(36) Hénin, J.; Chipot, C. Overcoming Free Energy Barriers Using Unconstrained Molecular Dynamics Simulations. J. Chem. Phys. 2004, 121 (7), 2904-2914

(37) Fiorin, G.; Klein, M. L.; Hénin, J. Using Collective Variables to Drive Molecular Dynamics Simulations. Mol. Phys. 2013, 111 (22-23), 3345-3362.

(38) Comer, J.; Phillips, J. C.; Schulten, K.; Chipot, C. Multiple-Replica Strategies for Free-Energy Calculations in NAMD: Multiple-Walker Adaptive Biasing Force and Walker Selection Rules. J. Chem. Theory Comput. 2014, 10 (12), 5276-5285.

(39) Minoukadeh, K.; Chipot, C.; Lelievre, T. Potential of Mean Force Calculations: A Multiple-Walker Adaptive Biasing Force Approach. J. Chem. Theory Comput. 2010, 6 (4), 1008-1017.

(40) Simpson, M. J.; Poblete, H.; Griffith, M.; Alarcon, E. I.; Scaiano, J. C. Impact of Dye-Protein Interaction and Silver Nanoparticles on Rose Bengal Photophysical Behavior and Protein Photocrosslinking. Photochem. Photobiol. 2013, 89 (6), 1433-1441.

(41) Xia, Y.; Xiong, Y.; Lim, B.; Skrabalak, S. E. Shape-Controlled Synthesis of Metal Nanocrystals: Simple Chemistry Meets Complex Physics? Angew. Chem. Int. Ed. 2009, 48 (1), 60-103.

(42) Wright, L. B.; Walsh, T. R. Efficient Conformational Sampling of Peptides Adsorbed onto Inorganic Surfaces: Insights from a Quartz Binding Peptide. Phys. Chem. Chem. Phys. PCCP 2013, 15 (13), 4715-4726.

(43) Hazel, A.; Chipot, C.; Gumbart, J. C. Thermodynamics of DecaAlanine Folding in Water. J. Chem. Theory Comput. 2014, 10 (7), 28362844.

(44) Szabo, A.; Schulten, K.; Schulten, Z. First Passage Time Approach to Diffusion Controlled Reactions. J. Chem. Phys. 1980, 72 (8), 4350-4357.

(45) Jorgensen, W. L.; Severance, D. L. Aromatic-Aromatic Interactions: Free Energy Profiles for the Benzene Dimer in Water, Chloroform, and Liquid Benzene. J. Am. Chem. Soc. 1990, 112 (12), 4768-4774. 